\title{
Guaranteed Cost Certification for Discrete-Time Linear Switched Systems with a Dwell Time
}

\author{
Marc Jungers and Jamal Daafouz, Member IEEE,
}

\begin{abstract}
This paper studies the guaranteed cost of a quadratic criterion associated with a linear discrete-time switched system for all the set of admissible switching laws. The admissible switching laws are here the ones exhibiting a dwell time. The approach provided here is to design an upper bound and a parametrized family of lower bounds of the guaranteed cost as close as possible in order to obtain a certification of the guaranteed cost. The upper bound is determined via a switched Lyapunov function and the lower bounds are obtained via the numerical computation of the cost induced by particular periodic switching laws. The features of the proposed approach are illustrated by a numerical example.
\end{abstract}

Index Terms-Switched systems; performance certification; periodic Lyapunov equations

\section{INTRODUCTION}

A linear switched system is an association of a set of a finite number of LTI dynamic systems and a switching law [1]. In practice, there are many applications where switched systems modelling is appropriate, like embedded systems in automotive industry, aerospace and energy management. The last decades have witnessed an increasing interest from the scientific community in the study of the stability property for this class of hybrid systems [1]-[4], by emphasizing tools among them Lyapunov functions depending on the switching parameter [5] or multiple Lyapunov functions [6], [7]. A situation which is often encountered in real applications is the one where a mode must remain

This work was partially supported by ANR project ArHyCo, Programme "Systèmes Embarqués et Grandes Infrastructures" ARPEGE, contract number ANR-2008 SEGI 004 01-30011459 and by the European Community's Seventh Framework Programme (FP7/2007-2013) under grant agreement $\mathrm{n}^{\circ}$ 257462: HYCON2 Network of Excellence "Highly-Complex and Networked Control Systems".

The authors are with Université de Lorraine, CRAN, UMR 7039, 2 avenue de la forêt de Haye, Vandœuvre-lès-Nancy Cedex, 54516, France and CNRS, CRAN, UMR 7039, France. J. Daafouz is also with Institut Universitaire de France.

Corresponding author: M. Jungers, marc.jungerseuniv-lorraine.fr active at least during a constant time interval called a dwell time.

Beyond questions of stability, the performance aspects of switched systems have been investigated and still remain an open issue [8], like the notion of the guaranteed cost of a performance index. Determining such an extremum over all the admissible switching laws is a difficult task, even if theoretical answers have been provided via hybrid versions of the Pontryagin Maximum Principle [9], [10], or dynamic programming [11], [12]. Another way to study the guaranteed cost of a performance index is to consider a set of its upper bounds and to select in this set the smallest value. Obtaining an upper bound of a guaranteed cost as small as possible does not provide information on its gap. This is the issue of the certification by exhibiting upper and lower bounds as close as possible.

This manuscript deals with the problem of the performance certification for a discrete-time linear switched system with a switching law under a dwell time assumption. The performance is chosen as the 2-norm of the output. Firstly the upper bound of the guaranteed cost will be designed by the help of the initial value of a switched Lyapunov function. Secondly the lower bounds are determined from the following observation: the exact value of the criterion associated with a particular admissible switching law is less than the guaranteed cost and is one of its lower bounds. Such a certification was firstly illustrated in [13], [14] by considering for the lower bounds the simple case of constant switching laws. Here we propose a less restrictive case using switching laws exhibiting the following specifications: being periodic and satisfying a dwell time. The exact value of the criterion, in this framework, is obtained via periodic Lyapunov equations.

The periodic Lyapunov equations have been studied [15], for both the theoretic (see for instance [16]-[19]) and numerical aspects [20], [21]. Here an algorithm based on the shift-invariant rep- 
resentation and adapted to include the dwell time as a parameter is proposed.

The paper is organized as follows. Section II gives the problem formulation. Section III provides the main results to design upper and lower bounds of the guaranteed cost by taking into account the dwell time. Section IV presents the certification of the guaranteed cost and offers computational methods for its obtaining. Section V emphasizes the efficiency of our certification on an academic example.

Notations. $\mathbb{R}, \mathbb{N}, \mathbb{C}$ are respectively the sets of real, natural and complex numbers and $\mathbb{N}^{*}=$ $\mathbb{N} /\{0\}$. If $\mathbb{S}$ is a set, $\mathbb{S}^{N}$ is the cartesian product $\mathbb{S} \times \cdots \times \mathbb{S}, N$ times. For two symmetric matrices, $A$ and $B, A>B$ means that $A-B$ is positive definite. $A^{\prime}$ denotes the transpose of $A$. The operator $\operatorname{diag}(A ; B)$ is a block diagonal matrix of $A$ and $B$.

\section{PROBlem DEFINITION}

Let us consider the autonomous linear switched system in discrete-time

$$
\begin{aligned}
x_{k+1} & =A_{\sigma(k)} x_{k}, \\
z_{k} & =C_{\sigma(k)} x_{k},
\end{aligned}
$$

where $x_{k} \in \mathbb{R}^{n}$ is the state of the system, $z_{k} \in \mathbb{R}^{p}$ the performance output and $\sigma \in \Sigma=\{\sigma: \mathbb{N} \rightarrow \mathcal{I}\}$, where $\mathcal{I}=\{1, \cdots, N\}$ is the switching rule which indicates the active mode at each time $k: A_{\sigma(k)}$ (respectively $C_{\sigma(k)}$ ) belonging to the finite set of matrices $\left\{A_{1}, \cdots, A_{N}\right\}$ (respectively $\left\{C_{1}, \cdots, C_{N}\right\}$ ). We assume that the switching law verifies a dwell time, defined as follows.

Definition 1: For an integer $\Delta \in \mathbb{N}^{*}$, the set of the switching laws satisfying a dwell time at least equal to $\Delta$ is defined by

$$
\mathcal{D}_{\Delta}=\left\{\sigma \in \Sigma ; \exists\left\{\ell_{q}\right\}_{q \in \mathbb{N}}, \ell_{q+1}-\ell_{q} \geq \Delta ;\right.
$$$$
\left.\sigma(k)=\sigma\left(\ell_{q}\right), \forall \ell_{q} \leq k<\ell_{q+1} ; \sigma\left(\ell_{q}\right) \neq \sigma\left(\ell_{q+1}\right)\right\} \text {. }
$$

The subsequence $\left\{\ell_{q}\right\}_{q \in \mathbb{N}}$, induced in $\mathcal{D}_{\Delta}$ consists in the switching times related to the switching law $\sigma$. Due to the definition of $\mathcal{D}_{\Delta}$ we have the following properties $\tilde{\Delta} \geq \Delta \Rightarrow \mathcal{D}_{\tilde{\Delta}} \subset \mathcal{D}_{\Delta}$.

In addition $\mathcal{D}_{1}=\Sigma$ is the set of arbitrary switching laws and $\mathcal{D}_{\infty}$ is the set of the $N$ constant switching laws. The performance of the system (1) is chosen as the norm of the performance output $z_{k}$ :

$$
J\left(\sigma, x_{0}\right)=\sum_{k=0}^{+\infty} z_{k}^{\prime} z_{k}=\sum_{k=0}^{+\infty} x_{k}^{\prime} C_{\sigma(k)}^{\prime} C_{\sigma(k)} x_{k}
$$

The aim of this note is to obtain an evaluation of the guaranteed cost,

$$
\gamma\left(x_{0}\right)=\sup _{\sigma \in \mathcal{D}_{\Delta}} J\left(\sigma, x_{0}\right),
$$

which is a quadratic form with respect to the initial state $x_{0}$.

Obtaining analytically the value of $\gamma\left(x_{0}\right)$ is not possible in general via the Pontryagin Maximum Principle or dynamic programming, even in the case of switched linear systems, due to the timedependency and the infinite time horizon of the criterion $J$. Moreover, one may apply dynamic programming over a truncated horizon but at the expense of a numerical explosion when increasing the size of the truncated horizon. The exact numerical computation of an upper bound and a parametrized family of lower bounds for particular switching laws is considered in this note.

\section{UPPER AND LOWER BOUNDS OF THE GUARANTEED COST}

This section is devoted to designing upper bounds and a family of lower bounds of $\gamma\left(x_{0}\right)$. The associated main results are given in Theorem 1 .

\section{A. Preliminaries and notations}

Preliminaries about tools for periodic systems [15], [17] are recalled here.

Definition 2: For $K \in \mathbb{N}^{*}$, the set of the $K$ periodic switching laws is defined by $\mathcal{P}_{K}=$ $\{\sigma \in \Sigma ; \sigma(k+K)=\sigma(k), \forall k \in \mathbb{N}\}$.

Definition 3: For $(\Delta, d) \in \mathbb{N}^{*} \times \mathbb{N}^{*}$, let us define

$$
\begin{gathered}
\mathcal{S}_{\Delta, d}=\{\sigma \in \Sigma ; \sigma(k+d \Delta)=\sigma(k), \forall k \in \mathbb{N}, \\
\sigma(i \Delta)=\sigma(i \Delta+j), \\
\forall(j, i) \in\{0, \cdots, \Delta-1\} \times\{0, \cdots, d-1\}\} .
\end{gathered}
$$

A switching law belonging to $\mathcal{S}_{\Delta, d}$ has a dwelltime equal to $\Delta$ and is $d \Delta$-periodic. $\mathcal{S}_{\Delta, d}$ is then a subset of $\mathcal{D}_{\Delta}$ and $\mathcal{P}_{d \Delta}$ and one gets $\mathcal{S}_{\Delta, d} \subset$ $\left(\mathcal{D}_{\Delta} \cap \mathcal{P}_{d \Delta}\right)$.

The set $\mathcal{S}_{\Delta, d}$ contains exactly $N^{d}$ switching laws, which are characterized by the $d$-uplet $\sigma_{d} \in \mathcal{I}^{d}$ defined by $\sigma_{d}=(\sigma(0), \sigma(\Delta), \cdots, \sigma((d-1) \Delta)$. We note $\pi$ the natural bijection allowing the link between $\mathcal{S}_{\Delta, d}$ and the cartesian product $\mathcal{I}^{d}$ by

$$
\pi:\left\{\begin{array}{lll}
\mathcal{I}^{d} & \longrightarrow & \mathcal{S}_{\Delta, d} \\
\left(i_{0}, i_{1}, \cdots, i_{d-1}\right) \longrightarrow & \sigma ; \sigma(q \Delta)=i_{q}, \\
& \forall q \in\{0, \cdots, d-1\} .
\end{array}\right.
$$


Definition 4: The state-transition matrix related to the system (1) is defined by $\forall i \in \mathbb{N}, \Phi_{i, i}=I_{n}$ and $\Phi_{i+j, i}=A_{\sigma(i+j-1)} A_{\sigma(i+j-2)} \cdots A_{\sigma(i)}, j \in \mathbb{N}^{*}$.

Definition 5: For $K \in \mathbb{N}^{*}$ and $\sigma \in \mathcal{P}_{K}$, the monodromy matrix at time $i$ is defined as $\Psi_{i}=\Phi_{i+K, i}$. The eigenvalues of $\Psi_{i}$ are called the characteristic multipliers of the system (1).

The characteristic multipliers of the system (1) are independent on the time $i$ (see [15, Chapter 3] for more details and a proof). The system (1) is globally exponentially stable if its characteristic multipliers belong strictly to the unit circle. The inclusion $\left\{A_{i}\right\}_{i \in \mathcal{I}}$ will be assumed here stable in order to have all the possible monodromy matrices stable and ensure the finiteness of $\gamma\left(x_{0}\right)$.

Definition 6: For $\sigma \in \mathcal{P}_{K}$, a characteristic multiplier of $A_{\sigma(\cdot)}$ is said to be $\left(A_{\sigma(\cdot)}, C_{\sigma(\cdot)}\right)$-observable at time $i$ if $\forall \lambda \in \mathbb{C}$

$$
\Psi_{i} x=\lambda x, C_{\sigma(k+i)} \Phi_{k+i, i} x=0,0 \leq \forall k \leq K-1
$$

implies $x=0$ or rank $\left[\begin{array}{c}\Psi_{i}-\lambda I_{n} \\ \mathcal{C}_{i, K, \sigma}\end{array}\right]=n, \forall \lambda \in \mathbb{C}$, where $\mathcal{C}_{i, K, \sigma}=\left[\begin{array}{c}C_{\sigma(i)} \\ C_{\sigma(i+1)} \Phi_{i+1, i} \\ \vdots \\ C_{\sigma(i+K-1)} \Phi_{i+K-1, i}\end{array}\right]$.

The pair $\left(A_{\sigma(\cdot)}, C_{\sigma(\cdot)}\right)$ is called observable if it is observable at any time $i, 0 \leq i \leq K-1$.

\section{B. Main results}

Theorem 1: For $(\Delta, d) \in \mathbb{N}^{*} \times \mathbb{N}^{*}$, let us consider the system (1)-(2) and the performance index $J$ defined by (3). Assume that for $\sigma \in \mathcal{D}_{\Delta}$, the pair $\left(A_{\sigma(\cdot)}, C_{\sigma(\cdot)}\right)$ is observable. If there exists symmetric positive definite matrices $P_{i} \in \mathbb{R}^{n \times n},(i \in \mathcal{I})$, symmetric positive definite matrices $W_{h} \in \mathbb{R}^{n \times n}$, $(h=0, \cdots, d-1)$ and a scalar $\beta_{\Delta}$ satisfying in one hand the LMIs, $\forall(i, j) \in \mathcal{I}^{2}, i \neq j$

$$
\begin{gathered}
P_{i}<\beta_{\Delta} I_{n}, \\
A_{i}^{\prime} P_{i} A_{i}-P_{i}+C_{i}^{\prime} C_{i}<0_{n}, \\
\left(A_{i}^{\prime}\right)^{\Delta} P_{j} A_{i}^{\Delta}-P_{i}+Q_{i}<0_{n},
\end{gathered}
$$

where $Q_{i}=\sum_{k=0}^{\Delta-1}\left(A_{i}^{\prime}\right)^{k} C_{i}^{\prime} C_{i} A_{i}^{k}, \forall i \in \mathcal{I}$ and in the other hand the equalities, for a $d$-uplet $\sigma_{d}=$ $\left(i_{0}, i_{1}, \cdots, i_{d-1}\right) \in \mathcal{I}^{d}, \forall \ell \in\{0, \cdots, d-2\}$,

$$
\left(A_{i_{d-1}}^{\prime}\right)^{\Delta} W_{0} A_{i_{d-1}}^{\Delta}-W_{d-1}+Q_{i_{d-1}}=0_{n},
$$

$$
\left(A_{i_{\ell}}^{\prime}\right)^{\Delta} W_{\ell+1} A_{i_{\ell}}^{\Delta}-W_{\ell}+Q_{i_{\ell}}=0_{n},
$$

then $J\left(\pi\left(\sigma_{d}\right), x_{0}\right)=x_{0}^{\prime} W_{0} x_{0}$, and for any $\sigma \in \mathcal{D}_{\Delta}$, the origin is globally exponentially stable and in addition the guaranteed cost $\gamma\left(x_{0}\right)$ is finite and verifies

$$
\begin{aligned}
& \gamma\left(x_{0}\right) \geq \bar{W}_{d}\left(x_{0}\right)=\max _{\sigma_{d} \in \mathcal{I}^{d}} J\left(\pi\left(\sigma_{d}\right), x_{0}\right) \\
& \gamma\left(x_{0}\right) \leq \bar{V}\left(x_{0}\right)=\max _{i \in \mathcal{I}} x_{0}^{\prime} P_{i} x_{0} \leq \beta_{\Delta}\left\|x_{0}\right\|^{2}
\end{aligned}
$$

where $\bar{V}\left(x_{0}\right)$ is an upper bound when $x_{0}$ is known, and $\beta_{\Delta}\left\|x_{0}\right\|^{2}$ when $x_{0}$ is unknown.

Proof: The proof is decomposed into two parts dedicated to the bounds (12) and (11).

The conditions (7) and (8) imply $A_{i}^{\prime} P_{i} A_{i}-P_{i}<$ $0_{n}$ and $\left(A_{i}^{\prime}\right)^{\Delta} P_{j} A_{i}^{\Delta}-P_{i}<0_{n}, \forall(i, j) \in \mathcal{I}^{2}, i \neq j$. These conditions are the same as those of [22, Theorem 1] leading to the global asymptotic stability of the origin. The relation (12) is proven in the same way of [22, Theorem 2], by noticing that $C_{\sigma(k)}^{\prime} C_{\sigma(k)}$ is constant between two consecutive switching times: $C_{\sigma(k)}^{\prime} C_{\sigma(k)}=C_{\sigma\left(\ell_{q}\right)}^{\prime} C_{\sigma\left(\ell_{q}\right)}, \forall \ell_{q} \leq$ $k<\ell_{q+1}$.

$$
\begin{array}{r}
A_{i}^{\prime}\left(P_{i}-Q_{i}\right) A_{i}-\left(P_{i}-Q_{i}\right)<-\sum_{k=1}^{\Delta}\left(A_{i}^{\prime}\right)^{k} C_{i}^{\prime} C_{i} A_{i}^{k} \\
-C_{i}^{\prime} C_{i}+\sum_{k=0}^{\Delta-1}\left(A_{i}^{\prime}\right)^{k} C_{i}^{\prime} C_{i} A_{i}^{k}<-\left(A_{i}^{\prime}\right)^{\Delta} C_{i}^{\prime} C_{i} A_{i}^{\Delta}<0_{n} .
\end{array}
$$

By induction, we infer that $\left(A_{i}^{\prime}\right)^{\tau}\left(P_{i}-Q_{i}\right) A_{i}^{\tau}<$ $\left(P_{i}-Q_{i}\right), \forall \tau \in \mathbb{N}^{*}$ and for any $\sigma \in \mathcal{D}_{\Delta}$,

$$
\begin{aligned}
& x_{\ell_{q+1}}^{\prime} P_{\sigma\left(\ell_{q+1}\right)} x_{\ell_{q+1}} \\
& \quad<x_{\ell_{q}}^{\prime}\left(A_{\sigma\left(\ell_{q}\right)}^{\ell_{q+1}-\ell_{q}-\Delta}\right)^{\prime}\left(P_{\sigma\left(\ell_{q}\right)}-Q_{\sigma\left(\ell_{q}\right)}\right) A_{\sigma\left(\ell_{q}\right)}^{\ell_{q+1}-\ell_{q}-\Delta} x_{\ell_{q}}, \\
& \quad<x_{\ell_{q}}^{\prime} P_{\sigma\left(\ell_{q}\right)} x_{\ell_{q}}-x_{\ell_{q}}^{\prime} Q_{\sigma\left(\ell_{q}\right)} x_{\ell_{q}} .
\end{aligned}
$$

By reordering the set $\mathbb{N}$ via the switching times: $\mathbb{N}=\cup_{q \in \mathbb{N}}\left\{\ell_{q}, \cdots, \ell_{q+1}-1\right\}$, we have

$$
\begin{aligned}
& J\left(\sigma, x_{0}\right)=\sum_{k=0}^{+\infty} x_{k}^{\prime} C_{\sigma(k)}^{\prime} C_{\sigma(k)} x_{k} \\
& =\sum_{q=0}^{+\infty} \sum_{j=0}^{\ell_{q+1}-\ell q-1} x_{\ell_{q}}^{\prime}\left(A_{\sigma\left(\ell_{q}\right)}^{j}\right)^{\prime} C_{\sigma\left(\ell_{q}\right)}^{\prime} C_{\sigma\left(\ell_{q}\right)} A_{\sigma\left(\ell_{q}\right)}^{j} x_{\ell_{q}} .
\end{aligned}
$$

By the definition of $Q_{i}$, one gets

$$
\begin{aligned}
Q_{\sigma\left(\ell_{q}\right)} & =\sum_{k=0}^{\Delta-1}\left(A_{\sigma\left(\ell_{q}\right)}^{k}\right)^{\prime} C_{\sigma\left(\ell_{q}\right)}^{\prime} C_{\sigma\left(\ell_{q}\right)} A_{\sigma\left(\ell_{q}\right)}^{k}, \\
& \geq \sum_{k=0}^{\ell_{q+1}-\ell q-1}\left(A_{\sigma\left(\ell_{q}\right)}^{k}\right)^{\prime} C_{\sigma\left(\ell_{q}\right)}^{\prime} C_{\sigma\left(\ell_{q}\right)} A_{\sigma\left(\ell_{q}\right)}^{k}
\end{aligned}
$$


because $\left(A_{\sigma\left(\ell_{q}\right)}^{k}\right)^{\prime} C_{\sigma\left(\ell_{q}\right)}^{\prime} C_{\sigma\left(\ell_{q}\right)} A_{\sigma\left(\ell_{q}\right)}^{k} \quad$ is positive semidefinite. With the help of inequality (13), the inequality $J\left(\sigma, x_{0}\right) \leq \sum_{q=0}^{+\infty} x_{\ell_{q}}^{\prime} Q_{\sigma\left(\ell_{q}\right)} x_{\ell_{q}}<$ $\sum_{q=0}^{+\infty}\left(x_{\ell_{q}}^{\prime} P_{\sigma\left(\ell_{q}\right)} x_{\ell_{q}}-x_{\ell_{q+1}}^{\prime} P_{\sigma\left(\ell_{q+1}\right)} x_{\ell_{q+1}}\right)$ holds.

Furthermore, it has been shown that the function $\left(x_{k}, \sigma(k)\right) \rightarrow x_{k}^{\prime} P_{\sigma(k)} x_{k}$ is a switched Lyapunov function for this system. Due to $\lim _{k \rightarrow+\infty} x_{k}^{\prime} P_{\sigma(k)} x_{k}=0$ and to the structure of a telescopic series, we have

$$
J\left(\sigma, x_{0}\right)<x_{0}^{\prime} P_{\sigma(0)} x_{0} .
$$

By multiplying the inequality (6) from the left by $x_{0}^{\prime}$ and from the right by $x_{0}$, we have $x_{0}^{\prime} P_{\sigma(0)} x_{0} \leq$ $\beta_{\Delta}\left\|x_{0}\right\|^{2}$. By taking the supremum over all $\sigma \in \mathcal{D}_{\Delta}$ of the inequality (14), we obtain the inequality (12).

The second part of the proof is based on the design of a $d \Delta$-periodic Lyapunov function associated with the $d \Delta$-periodic switching law $\sigma=\pi\left(\sigma_{d}\right)$, by the help of the symmetric positive definite matrices $W_{h}, h \in\{0, \cdots, d-1\}$.

Set $\tilde{P}_{0}=W_{0}>0_{n}$. Define $\tilde{P}_{d \Delta-1}$ by the equation (19), that is

$$
\begin{array}{r}
\tilde{P}_{d \Delta-1}=A_{\sigma(d \Delta-1)}^{\prime} \tilde{P}_{0} A_{\sigma(d \Delta-1)}+C_{\sigma(d \Delta-1)}^{\prime} C_{\sigma(d \Delta-1)} \\
=A_{i_{d-1}}^{\prime} \tilde{P}_{0} A_{i_{d-1}}+C_{i_{d-1}}^{\prime} C_{i_{d-1}}
\end{array}
$$

For $j \in\{2, \cdots, \Delta\}$, we set

$$
\begin{gathered}
\tilde{P}_{d \Delta-j}=A_{\sigma(d \Delta-j)}^{\prime} \tilde{P}_{d \Delta-j+1} A_{\sigma(d \Delta-j)} \\
+C_{\sigma(d \Delta-j)}^{\prime} C_{\sigma(d \Delta-j)}=\left(A_{i_{d-1}}^{j}\right)^{\prime} \tilde{P}_{0, \sigma_{d}} A_{i_{d-1}}^{j} \\
+\sum_{k=0}^{j-1}\left(A_{i_{d-1}}^{k}\right)^{\prime} C_{i_{d-1}}^{\prime} C_{i_{d-1}} A_{i_{d-1}}^{k}
\end{gathered}
$$

It is noteworthy that for $j=\Delta$, we have

$$
\begin{aligned}
& \tilde{P}_{(d-1) \Delta}=\left(A_{i_{d-1}}^{\Delta}\right)^{\prime} W_{0} A_{i_{d-1}}^{\Delta} \\
&+\sum_{k=0}^{\Delta-1}\left(A_{i_{d-1}}^{k}\right)^{\prime} C_{i_{d-1}}^{\prime} C_{i_{d-1}} A_{i_{d-1}}^{k} \\
&=\left(A_{i_{d-1}}^{\Delta}\right)^{\prime} W_{0} A_{i_{d-1}}^{\Delta}+Q_{i_{d-1}}=W_{d-1}>0_{n} .
\end{aligned}
$$

In the same way, it induces that $\tilde{P}_{0}=A_{i_{0}}^{\prime} \tilde{P}_{1} A_{i_{0}}+$ $C_{i_{0}}^{\prime} C_{i_{0}}=\left(A_{i_{0}}^{\prime}\right)^{\Delta} W_{1} A_{i_{0}}^{\Delta}+Q_{i_{0}}=W_{0}$, which proves that such a sequence $\left\{\tilde{P}_{\ell}\right\}_{\ell \in\{0, \ldots, d \Delta-1\}}$ is positive definite, symmetric and solves the periodic Lyapunov equation associated with $\sigma=\pi\left(\sigma_{d}\right) ; 0 \leq$ $\forall k \leq d \Delta-2$ :

$$
A_{\sigma(k)}^{\prime} \tilde{P}_{k+1} A_{\sigma(k)}-\tilde{P}_{k}+C_{\sigma(k)}^{\prime} C_{\sigma(k)}=0_{n} ;
$$

$A_{\sigma(K-1)}^{\prime} \tilde{P}_{0} A_{\sigma(K-1)}-\tilde{P}_{K-1}+C_{\sigma(K-1)}^{\prime} C_{\sigma(K-1)}=0_{n}$.

Due to inequalities (7) and (8), the system (1) is stable for any $\sigma \in \mathcal{D}_{\Delta}$, that is the characteristic multipliers belong strictly to the unit circle. The infinite series

$$
\begin{aligned}
\sum_{i=0}^{+\infty} \Phi_{\ell+i, \ell}^{\prime} C_{\sigma(\ell+i)}^{\prime} C_{\sigma(\ell+i)} \Phi_{\ell+i, \ell} & \\
& =\sum_{q=0}^{+\infty}\left(\Psi_{\ell}^{\prime}\right)^{q} \mathbb{C}_{\ell, K, \sigma}^{\prime} \mathbb{C}_{\ell, K, \sigma} \Psi_{\ell}^{q}, \quad \forall \ell \in \mathbb{N}
\end{aligned}
$$

are well defined, symmetric and solve the periodic Lyapunov equation (18)-(19), by arguments proposed in [16], [17], [23]. By invoking [17, Theorem 1], the solution of the periodic Lyapunov equation is unique. This implies that the infinite series, definied by (20) are equal to $\tilde{P}_{\ell}$. Because the pair $\left(A_{\sigma(\cdot)}, C_{\sigma(\cdot)}\right)$ is assumed observable, classical arguments allow to prove the positive-definitness of matrix $\tilde{P}_{\ell}$.

Let us prove now that the value of the criterion $J\left(\pi\left(\sigma_{d}\right), x_{0}\right)$ is given by $x_{0}^{\prime} \tilde{P}_{0} x_{0}=x_{0}^{\prime} W_{0} x_{0}$, because

$$
\sum_{k=0}^{+\infty} x_{0}^{\prime} \Phi_{k, 0}^{\prime} C_{\sigma(k)}^{\prime} C_{\sigma(k)} \Phi_{k, 0} x_{0}=x_{0}^{\prime} \tilde{P}_{0} x_{0} .
$$

The switching law $\pi(\sigma)$ belonging to $\mathcal{S}_{\Delta, d}$ which is a subset of $\mathcal{D}_{\Delta}$, we obtain the inequality (11), which ends the proof.

The main results proposed in Theorem 1 deserve some comments.

- The stability of $A_{i}$ and the observability of the $N$ pairs $\left(C_{i}, A_{i}\right), i \in \mathcal{I}$ are necessary conditions for the existence of symmetric positive definite matrices $P_{i}$ satisfying inequalities (7).

- The upper bound $\bar{V}\left(x_{0}\right)=\max _{i \in \mathcal{I}} x_{0}^{\prime} P_{i} x_{0}$ of $\gamma\left(x_{0}\right)$ is less than $\beta_{\Delta}\left\|x_{0}\right\|^{2}$ but depends on the direction of $x_{0}$ instead of $\beta_{\Delta}\left\|x_{0}\right\|^{2} . \beta_{\Delta}$ is the maximal eigenvalue of matrices $P_{i}$.

- For $\Delta=1$, we recover the method and the conditions proposed originally in [13], [14].

\section{CERTIFICATION AND NUMERICAL METHODS}

Theorem 1 offers tools to obtain classes of upper bounds (12) and lower bounds (11) of the guaranteed cost $\gamma\left(x_{0}\right)$. The smallest upper bound is given by the following optimization problem 
Optimization problem 1: Under the assumptions of Theorem 1, the smallest upper bound (12) is solution of

$$
\min _{P_{i}, \beta_{\Delta}} \beta_{\Delta}
$$

subject to the inequalities (6); (7) and (8).

The Theorem 1 provides a family of lower bounds, based on the solution of the $d \Delta$-periodic Lyapunov equation (18)-(19) parametrized by a $d$ uplet $\sigma_{d} \in \mathcal{I}^{d}$. The numerical computation of the lower bounds may be conveniently achieved by using a shift-invariant representation [20], [21], with a block-diagonal structure of the extended Lyapunov matrix. An optimization problem under linear matrix inequalities is then proposed to solve the periodic Lyapunov equations [24], when the symmetric positive definite solution exists and is unique. This allows to obtain numerically the lower bound $\bar{W}_{d}\left(x_{0}\right)$ defined by (11) and satisfying also $\bar{W}_{d}\left(x_{0}\right)=\max _{\sigma_{d} \in \mathcal{I}^{d}} x_{0}^{\prime} W_{0} x_{0}$, due to the fact that $W_{0}$ depends on the $d$-uplet $\sigma_{d}$. The numerical result is gathered in the optimization problem 2 . For $\sigma_{d}=\left(i_{0}, i_{1}, \cdots, i_{d-1}\right) \in \mathcal{I}^{d}$, we denote $\mathbb{W}_{d}=\operatorname{diag}\left(W_{0} ; W_{1} ; \cdots ; W_{d-1}\right) ; \mathbb{Q}_{\sigma_{d}}=$ $\operatorname{diag}\left(Q_{i_{0}} ; Q_{i_{1}} ; \cdots ; Q_{i_{d-1}}\right)$ and

$$
\mathbb{A}_{\Delta, \sigma_{d}}=\left[\begin{array}{cccc}
0_{n} & & & A_{i_{d-1}}^{\Delta} \\
A_{i_{0}}^{\Delta} & \ddots & & \\
& \ddots & \ddots & \\
& & A_{i_{d-2}}^{\Delta} & 0_{n}
\end{array}\right] .
$$

Optimization problem 2: For $(\Delta, d) \in \mathbb{N}^{*} \times \mathbb{N}^{*}$, consider the optimization variables as symmetric positive definite matrices $W_{i}, i=0, \cdots d-1$. If the system (1) is stable and if the pair $\left(A_{\sigma(\cdot)}, C_{\sigma(\cdot)}\right)$ is observable, then the optimization problem

$$
\begin{gathered}
\min _{\left\{W_{i}\right\}_{i \in\{0, \cdots, d-1\}}} \operatorname{Trace}\left(\mathbb{W}_{d}\right) \\
\text { subject to } \mathbb{W}_{d}=\mathbb{W}_{d}^{\prime}>0_{d n}, \text { and } \\
\mathbb{A}_{\Delta, \sigma_{d}}^{\prime} \mathbb{W}_{d} \mathbb{A}_{\Delta, \sigma_{d}}-\mathbb{W}_{d}+\mathbb{Q}_{\sigma_{d}} \leq 0_{d n},
\end{gathered}
$$

leads to the unique symmetric and positive solution of equations (9)-(10).

Moreover, it is possible to quantify more precisely the smallest gap between $\gamma\left(x_{0}\right)$ and $\bar{W}_{d}\left(x_{0}\right)$. Let introduce the scalar $\xi_{\Delta, d}=\sup _{x_{0},\left\|x_{0}\right\|=1} \bar{W}_{d}\left(x_{0}\right)=\max _{\sigma_{d} \in \mathcal{I}^{d}} \lambda_{\max }\left(W_{0}\right)$. By definition of $\beta_{\Delta}$ and $\xi_{\Delta, d}$, we have $\inf _{x_{0} \in \mathbb{R}^{n}}\left(\left(\bar{V}\left(x_{0}\right)-\bar{W}_{d}\left(x_{0}\right)\right) /\left\|x_{0}\right\|^{2}\right) \leq \beta_{\Delta}-\xi_{\Delta, d}$.

The certification of the guaranteed cost $\gamma\left(x_{0}\right)$ is especially good for an particular initial condition as $\xi_{\Delta, d}$ is close to $\beta_{\Delta}$. The decreasing inclusion of $\mathcal{D}_{\Delta}$ implies that $\beta_{\Delta}$ is a decreasing function of $\Delta$. Because $\beta_{\Delta} \geq 0$, we have $\lim _{\Delta \rightarrow \infty} \beta_{\Delta}=\beta_{\infty}$, where $\beta_{\infty}$ is the solution of the optimization problem 1 , without inequality (8) as a constraint.

In addition, when $d=1$, the periodic Lyapunov solutions are restricted to be constant and they depend only on the initial mode, we have thus $W_{0}=P_{\sigma(0)}=\tilde{P}_{\sigma(0)}$, for any dwell time $\Delta$. One gets $\xi_{\Delta, 1}=\beta_{\infty}$. Furthermore, if $d_{2}$ is a multiple of $d_{1}$, then $\mathcal{S}_{\Delta, d_{1}} \subset \mathcal{S}_{\Delta, d_{2}}$ which implies that $\xi_{\Delta, d_{1}} \leq \xi_{\Delta, d_{2}}$. Nevertheless, $\xi_{\Delta, d}$ is not an increasing function of $d$.

\section{ILLLUSTRATION}

Let us consider the next example, with $N=2$, $n=2, A_{1}=\left[\begin{array}{cc}0.9 & 0 \\ 0.1 & 0.8\end{array}\right], A_{2}=\left[\begin{array}{cc}0.9 & 0.5 \\ 0 & 0.8\end{array}\right]$, $C_{1}=I_{2}$ and $C_{2}=0.5 I_{2}$. We can check in Fig. 1 that $\beta_{\Delta}$ is a decreasing function of the dwell time, converging, as expected, to $\beta_{\infty}=7.07$.

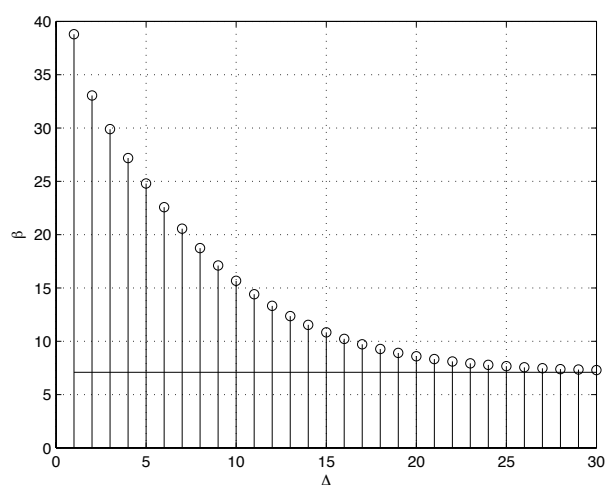

Fig. 1. Upper bound $\beta$ in function of the dwell time $\Delta$.

For a dwell time $\Delta$, we define $x_{0}=$ $(\cos (\alpha) \sin (\alpha))^{\prime}$, with $\alpha \in[0, \pi]$, because $J$ is quadratic. We consider here $\Delta=4$. The value of the upper bound $\bar{V}\left(x_{0}\right)$ and the lower bounds $\bar{W}_{d}\left(x_{0}\right)(d \in\{1, \cdots, 6\})$ are depicted on Fig. 2 in function of $\alpha$. It can be seen that the periodic behavior helps to take into account the evolution of the switched system and improves the lower bound. The values $\xi_{4, d}(d \in\{1, \cdots, 6\})$ are compared with $\beta_{4}=27.2$ in Tab. I. We could verify that $\xi_{4,3}<\xi_{4,2}$, that is $\xi_{4, d}$ is not an increasing function of $d$, but we have $\xi_{4,1}<\xi_{4,2}<\xi_{4,4}<\xi_{4,6}<\xi_{4,8}$ and $\xi_{4,1}<\xi_{4,3}<\xi_{4,6}$. The gap between the bounds is characterized by $\left(\beta_{4}-\xi_{4,2}\right) / \beta_{4}=3 \%$, which drastically improves the rate $\left(\beta_{4}-\xi_{4,1}\right) / \beta_{4}=71 \%$. 


\begin{tabular}{|c||c|c|c|c|c|c|}
\hline$d$ & 1 & 2 & 3 & 4 & 5 & 6 \\
\hline$\xi_{4, d}$ & 7.07 & 26.3 & 19.9 & 26.3 & 23.4 & 26.3 \\
\hline
\end{tabular}

\section{TABLE I}

VALUES $\xi_{4, d}$ IN FUNCTION OF THE PARAMETER $d$.

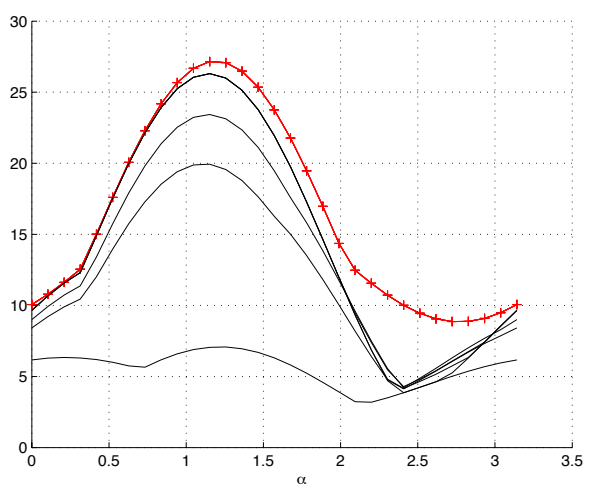

Fig. 2. For $\Delta=4$, bounds $\bar{V}\left(x_{0}\right)$ (depicted by '+-') and $\bar{W}_{d}\left(x_{0}\right)$ $(d \in\{1, \cdots, 9\}$, depicted by '-') in function of $\alpha$.

\section{CONCLUSION}

The performance certification of a quadratic cost associated with an autonomous linear discrete-time switched system for the class of switching laws with a dwell time has been studied. The certification proposed here consists in designing, for a dwell time, upper and lower bounds as close as possible. The presented upper bound is obtained via the initial value of a switched Lyapunov function taking into account the dwell time. A family of lower bounds is determined by computing the cost value for a specific periodic switching law. The algorithm used for this computation is based on solving a periodic Lyapunov equation with a period multiple of the dwell time. An academic example illustrates the features of our approach.

\section{REFERENCES}

[1] D. Liberzon, Switching in Systems and Control, ser. Systems and Control: Foundations and Applications. Birkhäuser, 2003.

[2] R. A. Decarlo, M. S. Branicky, S. Pettersson, and B. Lennartson, "Perspective and results on the stability and stabilization of hybrid systems." Proceedings of the IEEE, vol. 88, pp. 10691082, 2000.

[3] D. Liberzon and A. S. Morse, "Basic problems in stability and design of switched systems," IEEE Control Systems Magazine, vol. 19, pp. 59-70, 2003.

[4] R. Shorten, F. Wirth, O. Mason, K. Wulff, and C. King, "Stability criteria for switched and hybrid systems," SIAM Review, vol. 49, no. 7, pp. 545-592, 2007.
[5] J. Daafouz, P. Riedinger, and C. Iung, "Stability analysis and control synthesis for switched systems : A switched Lyapunov function approach," IEEE Trans. Automat. Control, vol. 47, pp. 1883-1887, 2002.

[6] M. S. Branicky, "Multiple Lyapunov functions and other analysis tools for switched and hybrid systems," IEEE Trans. Automat. Control, vol. 43, pp. 475-582, 1998.

[7] S. Pettersson and B. Lennartson, "LMI for stability and robustness for hybrid systems," in Proceedings of the American Control Conference, 1997, pp. 1714-1718.

[8] Z. Sun and S. S. Ge, "Analysis and synthesis of switched linear control systems," Automatica, vol. 41, pp. 181-195, 2005.

[9] B. Piccoli and H. J. Sussmann, "Regular synthesis and sufficiency conditions for optimality," SIAM J. Control and Optimization, vol. 39, no. 2, pp. 359-410, 2000.

[10] H. J. Sussmann, Nonlinear Control in the Year 2000. SpringerVerlag, 2000, ch. New Theories of Set-valued Differentials and New Versions of the Maximum Principle of Optimal Control Theory.

[11] V. Azhmyakov, R. G. Guerra, and M. Egerstedt, "Hybrid LQ-optimization using dynamic programming," in American Control Conference. Saint-Louis, MO, USA, June 2009.

[12] F. Borrelli, M. Baotić, A. Bemporad, and M. Morari, "Dynamic programming for constrained optimal control of discrete-time linear hybrid systems," Automatica, vol. 41, pp. 1709-1721, 2005.

[13] J. Melin, M. Jungers, J. Daafouz, and C. Iung, "Performance analysis and design of dynamic output feedback control for switched systems," International Journal of Control, vol. 84, no. 2, pp. 253-260, 2011.

[14] _ , "On analysis of performance for digitally controlled and time-varying delayed systems," in European Control Conference (ECC'09), Budapest, Hungary, 2009, pp. 4181-4186.

[15] S. Bittanti and P. Colaneri, Periodic Systems Filtering and Control. Springer, 2009.

[16] — - "Lyapunov and Riccati equations: periodic inertia theorems," IEEE Transactions on Automatic Control, vol. AC-31, no. 7, pp. 659-661, 1986.

[17] P. Bolzern and P. Colaneri, "The periodic Lyapunov equation," SIAM J. Matrix Anal. Appl., vol. 9, no. 4, pp. 499-512, 1988.

[18] S. Bittanti, P. Colaneri, and G. D. Nicolao, The Riccati equation. Springer-Verlag, 1991, ch. The periodic Riccati equation, pp. $127-162$.

[19] D. Kressner, "Large periodic Lyapunov equation: algorithms and applications," in European Control Conference (ECC'03), Cambridge, UK, 2003.

[20] B. Park and E. Verriest, "Canonical forms on discrete linear periodically time-varying systems and a control application," in Proceedings of the 28th IEEE Conference on Decision and Control, 1989, pp. 1220-1225.

[21] D. Flamm, "A new shift-invariant representation for periodic linear systems," in Proceedings of the American Control Conference, 1990, pp. 1510-1515.

[22] J. C. Geromel and P. Colaneri, "Stability and stabilization of discrete-time switched systems," International Journal of Control, vol. 79, no. 7, pp. 719-728, July 2006.

[23] H. Kano and T. Nishimura, "A note on periodic Lyapunov equations," in Proceedings of the 35th Conference on Decision and Control, 1996, pp. 35-36.

[24] F. A. Aliev and V. B. Larin, "Optimization problems for periodic systems," International Applied Mechanics, vol. 45, no. 11, pp. 1162-1188, 2009. 